SCIENTIFIC REPORT

\title{
Surgery for primary basal cell carcinoma including the eyelid margins with intraoperative frozen section control: comparative interventional study with a minimum clinical follow up of 5 years
}

\author{
R M Conway, S Themel, L M Holbach
}

Br J Ophthalmol 2004;88:236-238. doi: 10.1136/bjo.2003.025056

Aim: To assess recurrence of primary basal cell carcinoma (PBCC) including the eyelid margins after resection with or without intraoperative frozen section control (IFS).

Methods: Comparative non-randomised interventional study involving review of records of consecutive patients with histological diagnosis of PBCC including the eyelid margins, treated surgically at the University of Erlangen-Nürnberg between 1989 and 1998. Patients with a minimum clinical follow up of 5 years treated with (group I) or without (group II) IFS were compared. Postoperatively, permanent paraffin sections were available in all patients.

Results: 165 patients were available for study. There were 145 patients with a minimum of 5 years follow up. Of these, no tumour recurrences were observed in group I $(n=114)$ compared with three $(9.7 \%)$ in group II $(n=31)(p=0.002)$. Conclusions: Surgery for PBCC including the eyelid margins with IFS and immediate plastic reconstruction, as performed in the present study, is associated with better long term cure compared with surgery with clinical control.

B asal cell carcinomas (BCCs) of the eyelid are the commonest malignancy encountered in ophthalmic practice. ${ }^{12}$ Compared with primary basal cell carcinoma (PBCC), recurrent eyelid BCCs exhibit more aggressive biological behaviour associated with a less favourable prognosis and poorer outcome. ${ }^{12}$ Therefore, an important aspect in the successful management of eyelid BCC is ensuring complete tumour excision at the point of resection of the primary lesion. As BCCs, and especially those with an infiltrative component, commonly have a degree of subclinical spread it can be difficult to clinically assess the margins of the lesion at the time of excision.

A number of non-comparative case series have demonstrated that the use of intraoperative microscopic assessment of the surgical margins (such as the Mohs' technique or intraoperative frozen section (IFS) analysis) is reliable in ensuring complete microscopic BCC tumour excision and is associated with a low reported rate of recurrence. ${ }^{2-14}$ Such approaches allow objective feedback regarding the adequacy of excision while preserving as much healthy tissue as possible for plastic reconstruction. However, most studies to date have had a relatively short period of follow up $(<3$ years) which may be insufficient to detect late recurrences ( $>5$ years). ${ }^{2}{ }^{13} 15$ In addition, there have been no formal comparative studies to assess the incidence of eyelid BCC recurrence using intraoperative frozen section control compared with clinical control alone.
The aim of the present study was to undertake a comparative analysis with a minimum follow up of 5 years to determine the efficacy of resection and long term recurrence rate following excision of primary BCC of the eyelid using IFS control of the surgical margins compared with clinical control alone.

\section{PATIENTS AND METHODS}

The medical records of consecutive patients with a histological diagnosis of primary BCC of the eyelid treated surgically at the University of Erlangen-Nürnberg between 1989 and 1998 were reviewed. All lesions either involved or were situated close to (within $5 \mathrm{~mm}$ of) the lid margin (frank extramarginal lesions which could be resected without a full thickness lid excision involving the lid margin were not included in this study). Of these, patients with at least 5 years follow up involving review with slit lamp control and photographic documentation within the eye clinic of the University of Erlangen-Nürnberg, were identified.

From the patients with long term follow up ( $>5$ years), two study groups were defined. Group I included those patients who had undergone tumour excision with immediate plastic reconstruction using IFS. Group II included those patients who had undergone tumour excision and immediate plastic reconstruction using clinical control with the operating biomicroscope alone. All surgical specimens, including those used for IFS, were processed for postoperative paraffin sections.

The following clinicopathological variables were extracted from the medical records for each patient in the study groups: age, gender, race, anatomical localisation of the tumour, maximal tumour diameter determined clinically at the slit lamp (mm), histopathological classification (tumours were divided into two groups: (1) BCCs with an infiltrative growth pattern defined as a portion or all of the tumour consisting of elongated strands of infiltrating tumour cells (morphea and mixed BCCs); (2) those with a circumscribed growth pattern (nodular BCC), in which tumour cells grew in solid clumps or rounded cell nests), microscopic involvement of surgical margins by tumour cells determined by both frozen section and permanent paraffin section, recurrence after primary excision (recurrence was defined as the reappearance of a histologically confirmed BCC at the site of previous excision), time between primary surgery and diagnosis of BCC recurrence (years), and the period of follow up (years).

Abbreviations: $B C C$, basal cell carcinoma; IFS, intraoperative frozen section control; PBCC, primary basal cell carcinoma 
The surgical procedures were carried out as follows. For all cases, the tumour was excised with a margin of normal looking tissue determined by intraoperative biomicroscopy. In those cases in which IFS was undertaken, the normal tissue margin was 3-4 mm. In those cases which were managed without IFS, the normal tissue margin was 4$8 \mathrm{~mm}$. In group I patients, IFS analysis was carried out as previously described. ${ }^{14}$ In brief, frozen sections were examined from both the medial and lateral surgical margins and in some cases the deep surface, if there was clinical doubt regarding the adequacy of excision. The sectioning procedure was carried out by a trained pathology technician and the sections were assessed intraoperatively by the surgeon. In cases where the surgical margins were involved with tumour cells, further excision was undertaken and the procedure repeated until clear margins were obtained. All specimens were submitted for permanent paraffin sections. Group II patients with positive margins on permanent paraffin sections, no clinically evident residual tumour, and a circumscribed growth pattern were observed at 6 monthly intervals. Those with an infiltrative growth pattern or clinical evidence of residual disease were further excised. All operations were performed by one surgeon.

The clinicopathological features and incidence of BCC recurrence were compared between the two study groups using the four field $\chi^{2}$ test (significance level of 0.05 ) to assess for association between unpaired data.

\section{RESULTS}

A total of 165 consecutive patients with histologically confirmed primary eyelid BCC were identified. All patients were white and there was one tumour per patient. The right side was involved in $53.0 \%$. Of these, 145 patients had a minimum follow up of 5 years through the eye clinic, including 114 in group I and 31 in group II. Of the 20 patients $(12.1 \%)$ with less than 5 years follow up, we were able to ascertain that all had either died of non-BCC related causes or had moved outside the clinic catchment area by examination of the medical records and communication with primary care physicians

The clinicopathological features are summarised in table 1. With respect to the anatomical site of the tumours, a medial canthal location was slightly more common in group I patients. Involvement of other anatomical regions as well as histologic BCC type, in particular the presence of an infiltrative growth pattern, were not significantly different between the study groups. Final postoperative paraffin section control confirmed that the surgical margins were clear in all patients managed with the frozen section technique. In contrast, $9.7 \%$ of group II patients had positive margins on postoperative paraffin sections. None of the patients with positive margins had clinical evidence of residual tumour. Two of these patients had an infiltrative growth pattern on histopathology and were further excised, and the other patient was observed. No recurrences occurred in group I patients, while there were three $(9.7 \%)$ in group II $(p=0.002)$. The mean time between the excision of the primary lesion and the diagnosis of the recurrence was 5.1 years (range 1.9-10.6). On histopathology, all recurrent lesions displayed features of an infiltrative growth pattern.

\section{DISCUSSION}

The present study is the only comparative analysis demonstrating significantly reduced long term recurrence using IFS compared with clinical control for the management of primary periocular BCC involving the eyelid margins. All patients treated with IFS had tumour free margins on postoperative histology with no recurrences observed, compared with $9.7 \%$ positive tumour margins and three $(9.7 \%)$ recurrences in the group treated using clinical control. These data suggest that IFS, as used here, appears reliable in ensuring complete tumour removal and the low recurrence rate is comparable with Mohs surgery. ${ }^{1}$

$\mathrm{BCC}$ recurrences three years and longer after excision have been observed in this and other studies and highlight the need for long term follow up in defining cure. ${ }^{13}{ }^{15}$ Most previous studies evaluating eyelid BCC resection with intraoperative microscopic control have been non-comparative with considerable variability in follow up (between 2 months and 4 years). ${ }^{2-14}$ Only two studies investigating recurrence of excised primary BCC with a clearly defined minimum of 5 years follow up have been reported. Both series were non-comparative. One reported recurrences in $0.6 \%$ with Mohs' technique. ${ }^{7}$ The other, using IFS, reported

Table 1 Clinicopathological characteristics of the study groups

\begin{tabular}{|c|c|c|c|}
\hline Characteristic & Group I ( $n=114)$ & Group II $(n=31)$ & p Value \\
\hline Mean age (range) (years) & $71.5(44-94)$ & $65.9(45-91)$ & N/A \\
\hline Female:male ratio & $1.0: 0.9$ & 1.0:1.3 & $\mathrm{N} / \mathrm{A}$ \\
\hline \multicolumn{4}{|l|}{ Anatomical location, n (\%) } \\
\hline Medial canthal region* & $55(48.2)$ & $11(35.5)$ & $\mathrm{p}<0.05$ \\
\hline Lower lid, central & $33(28.9)$ & $10(32.2)$ & $p>0.05$ \\
\hline Lateral canthal region $\dagger$ & 20 (17.5) & $7(22.6)$ & $p>0.05$ \\
\hline Lower lid, entire lid & $5(4.5)$ & $3(9.7)$ & $p>0.05$ \\
\hline Upper lid, central & $1(0.9)$ & $0(0.0)$ & $p>0.05$ \\
\hline Mean tumour size (range) (mm)‡ & $13.3(2-32)$ & $12.9(5-18)$ & $\mathrm{N} / \mathrm{A}$ \\
\hline \multicolumn{4}{|l|}{ Histopathology§ } \\
\hline Infiltrative growth pattern, $\mathrm{n}(\%)$ & $24(21.0)$ & $7(22.6)$ & $p>0.05$ \\
\hline Circumscribed growth pattern, $\mathrm{n}(\%)$ & $90(79.0)$ & $24(77.4)$ & $p>0.05$ \\
\hline \multicolumn{4}{|l|}{ Histopathological margins } \\
\hline Positive margins on PPS, $\mathrm{n}(\%)$ & $0(0.0)$ & $3(9.7)$ & $p=0.002$ \\
\hline Follow up and recurrence rate & & & $p=0.002$ \\
\hline Mean follow up period (range) (years) & $6.4(5.0-11.1)$ & $6.9(5.0-14.1)$ & \\
\hline Recurrences after resection, $\mathrm{n}(\%)$ & $0(0.0)$ & $3(9.7)$ & \\
\hline Mean time to recurrence (range) (years) & N/A & $5.1(1.9-10.6)$ & \\
\hline \multicolumn{4}{|c|}{$\begin{array}{l}\text { *Defined as involvement of the medial quarter of the upper or lower lid and/or the angle. } \\
\text { †Defined as involvement of the lateral quarter of the upper or lower lid and/or the angle. } \\
\text { tRefers to maximal tumour diameter. } \\
\text { \$lnfiltrative growth component was defined as a portion or all of the tumour consisting of elongated strands of } \\
\text { infiltrating BCC tumour cells. } \\
\text { †Positive margins were defined as the presence of tumour cells at one or more cut surfaces of the surgical } \\
\text { specimen. } \\
\mathrm{N} \text {, number; N/A, not applicable; PPS, postoperative paraffin section. }\end{array}$} \\
\hline
\end{tabular}


recurrences in $2.2 \% .^{13}$ In contrast to these studies, in which follow up included either telephoning patients or was undefined, we believe the data in the present analysis are superior as only patients who had been followed up with slit lamp photo documentation through the university eye clinic were included. This represents a follow up rate of $87.9 \%$ ( 145 of 165 patients). Of the 20 patients with less than 5 years follow up, all had either died of unrelated causes or moved out of the area.

Although non-randomised, the study groups were similar with respect to reported factors influencing BCC recurrence including age, tumour size, invasive growth pattern, and immune status (there were no immunocompromised patients in this series). ${ }^{216}$ Only tumour location differed slightly between the study groups. Previous studies have noted a moderately increased recurrence risk associated with a medial canthal location, possibly related to more cautious excision or earlier deep extension. ${ }^{217}$ Despite the higher incidence of this location in group I patients, the results indicate that primary eyelid BCC in this area can be treated with microscopic control producing good results.

Frozen section analysis was carried out using a technique previously described by ourselves and others. ${ }^{13}{ }^{14}$ Although sampling of the margins using this technique is less comprehensive than Mohs surgery, in the context of resections involving the eyelid margin, where there are only two, or occasionally three (if the deep surface is included), margins which need to be examined, the findings of this study indicate that the results of IFS are comparable with Mohs surgery when performed by a collaborative surgeon/ pathology team. ${ }^{13}$ For elliptical extramarginal lesions, especially those with an increased risk of recurrence such as large and/or recurrent medial canthal lesions, the Mohs' technique, examining the entire surgical margin, may be a better option, even though it is more time consuming. ${ }^{1}$ In optimising the IFS procedure, we believe there are a number of critical factors. The surgeon needs to be closely involved and should determine the critical surgical margins for assessment (in this study, the margins were evaluated by the surgeon in the operating room with the assistance of a pathology technician trained in frozen section technique). The technical quality of the morphological details should be high, to facilitate accurate judgement of the margins. Finally, there needs to be close communication between the surgeon and IFS team, to allow continuous feedback and timely progression of the operation schedule.

\section{ACKNOWLEDGEMENTS}

The study was supported in part by the Alexander von Humboldt Foundation, Bonn, Germany (RMC), Förderverein Augenheilkunde,
Erlangen, Germany (LH), The Royal Australian and New Zealand College of Ophthalmologists and Sydney Eye Hospital (RMC).

\section{Authors' affiliations}

R M Conway, S Themel, L M Holbach, Department of Ophthalmology and Eye Hospital, University of Erlangen-Nürnberg, Erlangen, Germany R M Conway, Alexander von Humboldt, Fellow and Lecturer, Save Sight Institute, University of Sydney, Australia

This study was conducted according to the Ethical Guidelines of the University of Erlangen-Nürnberg Human Ethics Committee and followed the tenets of the Declaration of Helsinki.

Correspondence to: $\operatorname{Dr}$ R M Conway, Department of Ophthalmology and Eye Hospital, University Erlangen-Nürnberg, Schwabachanlage 6 , D-91054 Erlangen, Germany; rmaxconway@hotmail.com

Accepted for publication 16 June 2003

\section{REFERENCES}

1 Cook BE Jr, Bartley GB. Treatment options and future prospects for the management of eyelid malignancies: an evidence-based update. Ophthalmology 2001;108:2088-98.

2 Margo CE, Waltz K. Basal cell carcinoma of the eyelid and periocular skin. Surv Ophthalmol 1993;38:169-92

3 Payne JW, Duke JR, Butner R, et al. Basal cell carcinoma of the eyelids. A long-term follow-up study. Arch Ophthalmol 1969;81:553-8.

4 Chalfin J, Putterman AM. Frozen section control in the surgery of basal cell carcinoma of the eyelid. Am J Ophthalmol 1979:87:802-9.

5 Riefkohl R, Pollack S, Georgiade GS. A rationale for the treatment of difficult basal cell and squamous cell carcinomas of the skin. Ann Plast Surg 1985; 15:99-104.

6 Robins P, Rodriguez-Sains R, Rabinovitz H, et al. Mohs surgery for periocular basal cell carcinomas. J Dermatol Surg Oncol 1985;11:1203-7.

7 Mohs FE. Micrographic surgery for the microscopically controlled excision of eyelid cancers. Arch Ophthalmol 1986;104:901-9.

8 Callahan MA, Monheit G, Callahan A. Twelve years' experience with the Mohs-Tromovitch technique of skin cancer removal. Int Ophthalmol Clin 1989;29:247-51

9 Frank HJ. Frozen section control of excision of eyelid basal cell carcinomas: 8 1/2 years' experience. Br J Ophthalmol 1989;73:328-32.

10 Downes RN, Walker NP, Collin JR. Micrographic (MOHS') surgery in the management of periocular basal cell epitheliomas. Eye 1990:4:160-8.

11 Glatt HJ, Olson JJ, Putterman AM. Conventional frozen sections in periocular basal-cell carcinoma: a review of 236 cases. Ophthalmic Surg 1992;23:6-8.

12 Arlette JP, Carruthers A, Threlfall WJ, et al. Basal cell carcinoma of the periocular region. J Cutan Med Surg 1998;2:205-8.

13 Wong VA, Marshall JA, Whitehead KJ, et al. Management of periocular basal cell carcinoma with modified en face frozen section controlled excision. Ophthal Plast Reconstr Surg 2002;18:430-5.

14 Holbach LM, Jünemann A, Muhammad S. Surgical management of periocular basal cell carcinoma using frozen section control and immediate reconstruction-indications and methods in 106 patients. Klin Monatsb/ Augenheilkd 1998;213:278-83.

15 Steinkogler FJ, Scholda CD. The necessity of long-term follow up after surgery for basal cell carcinomas of the eyelid. Ophthalmic Surg 1993;24:755-8.

16 Batra RS, Kelley LC. Predictors of extensive subclinical spread in nonmelanoma skin cancer treated with Mohs micrographic surgery. Arch Dermatol 2002; 138:1043-51.

17 Pieh S, Kuchar A, Novak $P$, et al. Long-term results after surgical basal cell carcinoma excision in the eyelid region. Br J Ophthalmol 1999:83:85-8. 